\title{
Endovascular treatment of intracranial aneurysms: A single center experience
}

\author{
(D) Gökhan Yüce, (1) Mustafa Taşar
}

University of Health Sciences Turkey, Gülhane Training and Research Hospital, Clinic of Radiology, Ankara, Turkey

\section{Date submitted:}

16.03.2020

Date accepted:

12.06.2020

Online publication date: 15.09.2020

\section{Corresponding Author:}

Gökhan Yüce MD, University of Health Sciences Turkey, Gülhane Training and Research Hospital, Clinic of Radiology, Ankara, Turkey drgkhann85@gmail.com

ORCID:

orcid.org/0000-0003-3546-7965

Keywords: Intracerebral aneurysms, endovascular treatment, detachable coil, flow diverter stent

\begin{abstract}
Aims: Intracranial aneurysms (IA) are vascular anomalies that are mostly detected by life threatening complications such as intracerebral hematoma or subarachnoid hemorrhage. Recently endovascular treatments have become the major treatment modality. In this study, our objectives were to describe the clinical data of the patients with treated IAs, to evaluate the success rate of two treatment modalities [coil embolization and flow diverter stents (FDS)], and to document common complications.

Methods: The patients who were admitted to our tertiary center and underwent endovascular treatment (with coil embolization/FDS) in the interventional radiology unit between December 2018 and October 2019 were enrolled. The clinical data of the patients including aneurysm size, subtype, location, treatment modality, and pre and post procedural complications were analyzed retrospectively.
\end{abstract}

Results: Eighteen of 37 patients (48.6\%) were male and 19 (51.3\%) were female. The median age was 54 (minimum-maximum; 12-77) years. There was no predisposing factor in the clinical history except four patients. Regarding the aneurysm subtypes, 89\% of IAs were saccular and $11 \%$ were fusiform. The most common localization was internal carotid artery (48\%). Ten patients were admitted with ruptured aneurysm and twenty seven were asymptomatic and unruptured. Four patients died in the mean follow-up of nine months. Two patients had intervention-related complication (hemiparesis and hydrocephalus).

Conclusions: IAs can be effectively treated with endovascular techniques since cure rates are high and complications rates are reasonably low. Coil, FDS or both can be used in selected cases of ruptured or unruptured aneurysms.

\section{Introduction}

Intracranial aneurysms (IA) have been the focus of attention for both clinicians and experimental studies since they have versatile properties. IAs are vascular anomalies that are mostly detected with complications such as intracerebral hematoma, subarachnoid hemorrhage (SAH) or hydrocephalus. Mortality rate of IA complications have decreased from $30-33 \%$ to $10 \%$ in the last decade indicating that important and rapid steps have been taken in the field of treatment. Although surgical clipping was the gold standard treatment method in the past, endovascular treatment became an alternative to surgical treatment with the development of coil systems $(1,2)$.

Endovascular treatment of IA started with the use of latex balloons and pushable coils. However, their uses were associated with significant complications which leaded to the development of detachable coil systems (3). In aneurysms treated by the detachable coils, despite total occlusion, relapse may develop due to mechanical failure or re-growth of the residual neck $(3,4)$. Another method used in endovascular therapy is flow diverter stents (FDS). FDSs decrease the blood flow, change the transmural gradient of the vessel wall, and induce intra-aneurysmal thrombosis (5). Unlike the coated stents, the pores in the FDS allow the passage of adequate amounts of blood and by that way, the main artery line along with the thin perforating branches remains open (6). Although the introduction of this device is relatively new, experience is rapidly increasing. However, the choice of the best endovascular procedure and indications for the use of FDSs are still a matter of debate and deserve to be discussed.

${ }^{\circ}$ Copyright 2020 by the University of Health Sciences Turkey, Gülhane Faculty of Medicine / Gülhane Medical Journal published by Galenos Publishing House. 
There are previous reports in the literature but few of them comprehensively investigated the clinical and technical aspects of using those two separate techniques. The clinical properties of patients, selected treatments and technical difficulties may vary among centers according to the expertise of the specialists and interpretation of local or international guidelines.

Herein this study, our purpose was to document our single center experience in endovascular treatment of IAs. Our primary objectives were to describe the demographic and clinical data of the patients with IA (aneurysm subtypes, locations and size), to evaluate the success rate of two treatment modalities (coil embolization and FDS), and also to document the complications that we came across in the routine clinical practice.

\section{Methods}

In this study, the patients who were admitted to our tertiary center's neurosurgery department and underwent endovascular treatment (with coil embolization/FDS) in the interventional radiology unit between December 2018 and October 2019 were enrolled. The demographic and clinical data together with the images of the procedures were analyzed retrospectively. Local Ethics Committee of University of Health Sciences Turkey, Gülhane Training and Research Hospital approved the study protocol (approval number: 2020-59) in accordance with the principles of the Declaration of Helsinki.

Diagnostic digital subtraction angiography (DSA) (Infinix, Canon Medical Systems, Japan) was performed in all patients when they applied to our interventional radiology division. Routine blood tests, chest radiography and electrocardiography had been performed for each patient before the intervention and data were recorded retrospectively from the patient files. In our center, the routine clinical practice based on the clinical guidelines is to start dual antiplatelet therapy, including acetylsalicylic acid (100 mg-once a day) and clopidogrel (75 mg-once a day) at least five days before the procedure for patients who are planned to undergo FDS. Endovascular embolization of aneurysms was performed using detachable coils (Axium, Meditronics, Ireland) and stents (FRED ${ }^{\circledR}$, Tokyo, Japan) in the DSA system. The procedure was performed under general anesthesia in all patients. According to the aneurysm localization, after the catheterization of the vertebral or internal carotid artery, the appropriate working position was determined and a 'road map' was made by contrast agent injection. Then, the desired area was reached through the catheter by the help of the appropriate length microcatheter (Headway microvention, California, USA) guidewires. Varying number of coils [Axium Prime coils (extra soft), Meditronics, Ireland] and/or stents were used, depending on the size and morphology of the aneurysms.

\section{Statistical Analysis}

The Statistical Package for the Social Sciences, version 25 (Inc., Chicago, IL, USA) was used for the statistical analysis.
Descriptive analysis was made to identify the baseline characteristics of the patients. Mean, standard deviation, and range were used to describe continuous variables. Categorical variables were described as number and percentage. Median was used to describe non-parametric variables.

\section{Results}

Eighteen of 37 patients (48.6\%) were male and 19 (51.3\%) were female. The median age was 54 years (minimummaximum; 12-77). In 33 of 37 patients (89.1\%), there was no predisposing factor for IA in the clinical history. Ten patients had ruptured whereas twenty seven had unruptured IA. All patients with unruptured $I A$, who had no vasospasm during the procedure, were followed up in the neurosurgery intensive care unit in the post intervention period until they were clinically stabilized. They were discharged provided that no interventionrelated complication developed in three days. The patients with ruptured IA were intubated and sedated until the intracranial pressure returned to normal and the neurological condition began to improve. All patients with ruptured IA received nimodipine treatment in order to reduce vasospasm $(10 \mathrm{mg} / 24$ hours intravenous then $6 \times 60 \mathrm{mg}$ oral for 21 days).

When aneurysm subtypes were evaluated, $89 \%$ of IAs (33 patients) were saccular and $11 \%$ (four patients) were fusiform (Table 1). The mean aneurysm size was $6.4 \pm 2.9 \mathrm{~mm}$ (minimum and maximum sizes were 3.2 and $15 \mathrm{~mm}$, respectively). Among four patients with fusiform aneurysms, one was a 22-year-old male patient with a history of skull base fracture that had been diagnosed after a traffic accident that occurred two months before the admission and had required hospitalization for few weeks. The second patient had a $2.5 \mathrm{~cm}$ sized pseudoaneurysm detected in the cavernous segment of left internal carotid artery, which was diagnosed two months after a cranial trauma (Figure 1). The third patient with fusiform aneurysm was a 12-year-old male patient who had a history of transcranial operation for craniopharyngioma two years before the admission with IA. In the fourth patient, fusiform enlargement was detected in the supraclinoid segment of the right internal carotid artery during preoperative evaluation for recurrent bleeding.

Regarding the localizations of aneurysms, internal carotid artery aneurysms constituted the most common type (48.6\%). The least common localizations were the posterior cerebral artery and vertebral artery aneurysms. Ten (27\%) of the patients were admitted to neurosurgery with ruptured aneurysm whereas $27(83 \%)$ patients were admitted with an unruptured aneurysm (Table 1).

In 32 of 37 (86.4\%) patients, endovascular treatment was successfully performed without any significant complication. Detachable coil was placed in 16 and FDS was used in 19 patients whereas two patients required both (Table 1). The recorded indication for FDS was mainly treating complex wide 
necked saccular or fusiform IA. Four patients (10.8\%) died due to vasospasm and infection. In a patient with a wide-necked aneurysm in the M2 segment of the middle cerebral artery, branch loss occurred after implanting FDS. That patient had left hemiparesis in the postoperative period and was referred to the physical therapy center after discharge. In another patient who was admitted with a ruptured aneurysm in the M2 segment of the middle cerebral artery and underwent coil embolization, hydrocephalus findings (neurological worsening, severe enlargement in the $3^{\text {rd }}$ ventricle and lateral ventricles in the brain tomography) appeared two days after the treatment. That patient required external ventricular drainage which was removed after clinical and radiological improvement on the postoperative $7^{\text {th }}$ day. In a patient who had asymptomatic non-ruptured left internal carotid artery supraclinoid segment aneurysm, FDS was implanted. Fifteen days after the stent implantation, the patient was readmitted with an intraparenchymal hematoma in the left parietooccipital region. During the follow-up period, the patient died due to secondary infections. Endovascular treatments of two cases were shown on Figure 1 and 2.

Of all patients, only two had a prior history of endovascular treatment. The indication for the second intervention was filling of the aneurysm sac in both. Both FDS and coil embolization were performed in one patient as redo intervention, and only FDS was installed in the other.

\section{Discussion}

IA mostly present with complications or can be completely asymptomatic. Therefore, their actual incidence can be difficult to determine. In the study by Meyer et al. (7), rupture rate was reported as $1-8 \%$. SAH is the most common clinical presentation form of IA and it is an important health problem due to its high mortality and morbidity (8). In our study, ten of thirty seven patients were admitted with ruptured and complicated IA.

\begin{tabular}{|c|c|c|c|}
\hline Age years (median; minimum-maximum) & \multicolumn{3}{|l|}{$54(12-77)$} \\
\hline Aneurysm size mm (mean $\pm S D)$ & \multicolumn{3}{|l|}{$6.4 \pm 2.9$} \\
\hline Aneurysm subtype (number) & \multicolumn{3}{|l|}{$\begin{array}{l}\text { Saccular: } 33 \\
\text { Fusiform: } 4\end{array}$} \\
\hline Aneurysm localization (number/\%) & \multicolumn{3}{|c|}{$\begin{array}{l}\text { ICA-supraclinoid } 12(32.4 \%) \\
\text { ICA-cavernous } 6(16.2 \%) \\
\text { MCA-M1 segment } 4(10.8) \\
\text { MCA-bifurcation } 2(5.4 \%) \\
\text { MCA-M2 segment } 2(5.4 \%) \\
\text { Anterior communicating artery } 9(24.3 \%) \\
\text { Vertebral artery } 1(2.7 \%) \\
\text { Posterior cerebral artery } 1(2.7 \%)\end{array}$} \\
\hline Unruptured/ruptured aneurysm (number) & \multicolumn{3}{|c|}{$27 / 10$} \\
\hline Treatment modality, complication and exitus (number) & $\begin{array}{l}\text { Modality } \\
\text { Coil only } 16 \\
\text { FDS only } 19 \\
\text { Coil+FDS } 2\end{array}$ & $\begin{array}{l}\text { Complication } \\
1 \text { Hydrocephalus } \\
1 \text { Hemiparesis } \\
\text { None }\end{array}$ & $\begin{array}{l}\text { Exitus } \\
3 \\
1 \\
0\end{array}$ \\
\hline
\end{tabular}
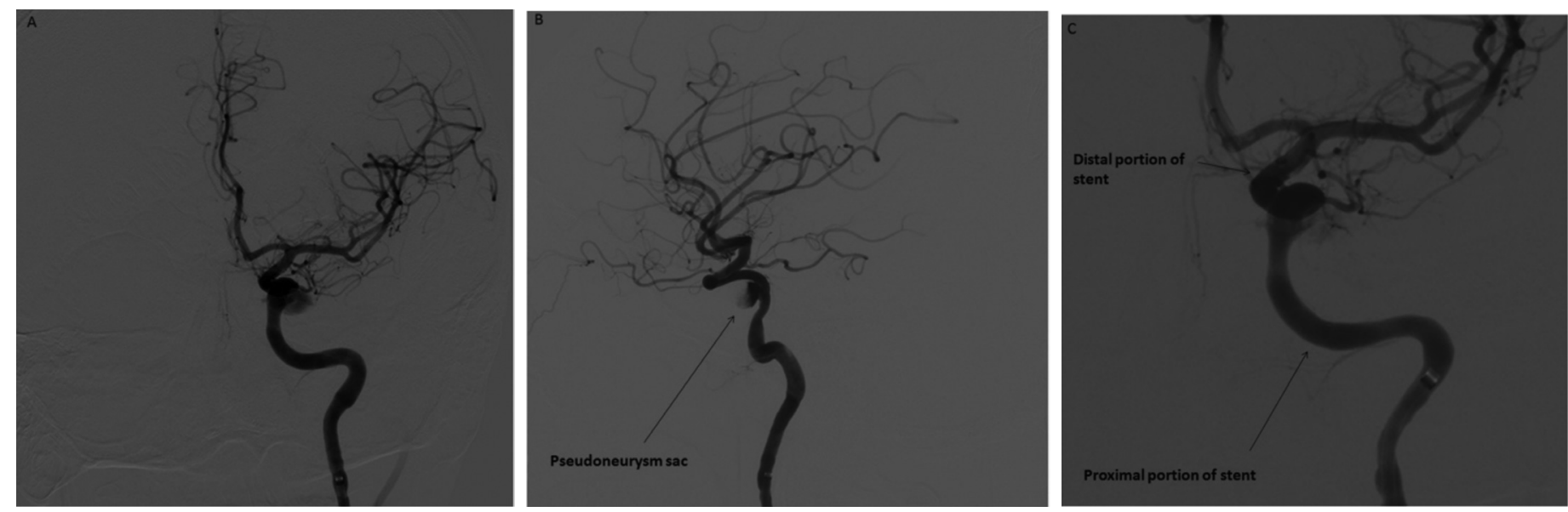

Figure 1. Preoperative lateral (a) and anteroposterior (b) angiographic image of a wide neck internal carotid artery aneurysm and post interventional (c) angiographic image shows stagnation of contrast agent within the aneurysm sac after stent replacement 

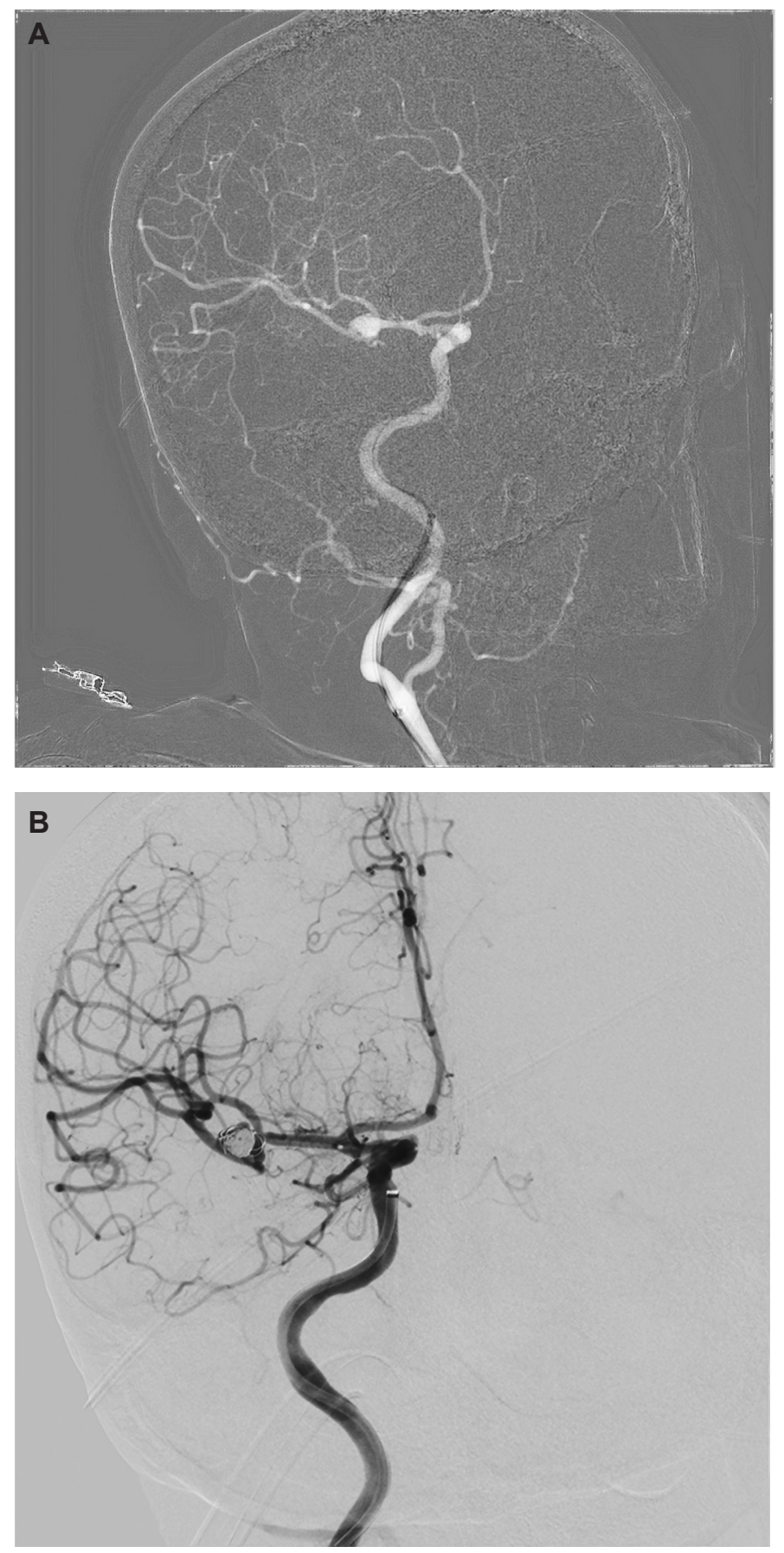

Figure 2. Preoperative angiographic image of a saccular aneurysm in the right MCA superior-inferior truncus bifurcation (a) angiographic image after coil embolization shows the effective volume reduction of the aneurysm sac (b)

IA can be classified as saccular (berry) or non-saccular. Saccular aneurysm, which is the most common type, looks like sacs or berries sticking out of a side of a blood vessel wall. Most of these saccular aneurysms have a "neck" region that may or may not be visualized during surgery $(9,10)$. Saccular aneurysms are associated with growth and rupture. The "fusiform" (dissecting) aneurysm is less common than the saccular aneurysm and looks like the blood vessel is expanded in all directions $(11,12)$. In our study, $89 \%$ of IAs (33 patients) were saccular and $11 \%$ (four patients) were fusiform. All patients with fusiform subtype had a predisposing factor such as cranial operation or previous trauma.

Coiling or clipping are accepted treatment options for endovascular treatment of both ruptured and unruptured aneurysm. After coiling or stent implantation, only $22-36 \%$ of the aneurysm volume can be filled (13). Two factors, which are the aneurysm size and neck width, determine the degree of volume reduction. There are publications indicating that endovascular treatment is less effective in wide-necked and giant aneurysms (14). In our study, 26 of the IAs were wide-necked. Three of four patients who died after endovascular treatment had widenecked aneurysms. Moreover, two patients who required recurrent intervention also had wide-necked aneurysms. Those findings support the previous reports indicating that prognosis gets worse as the neck widens. In our study group, the mean aneurysm size was $6.4 \mathrm{~mm}$. Ten patients had small-sized aneurysm ( $<4 \mathrm{~mm}), 22$ had medium-sized $(4-10 \mathrm{~mm})$ and 5 had large aneurysms (>10 mm).

The possible complications of coil embolization method were the need for recanalization and coil compaction in large series $(14,15)$. The rate of recanalization was reported as $33.6 \%$ and the rate of re-treatments was $20.7 \%$ in the study of Raymond et al. (15) including 383 aneurysms. In that aforementioned study, it was stated that the rate of contrast filling and baseline aneurysm size greater than $10 \mathrm{~mm}$ were the major determinants of recurrence after endovascular coil embolization. In our study, coil embolization was performed in 18 of 37 aneurysms (16 patients underwent only coil placement whereas 2 had both coil and FDS). During the median follow-up of six months (minimummaximum; 4-15 months), none of the aneurysms had contrast filling within the neck.

FDS have greatly changed the landscape of IA therapy and are now considered first-line therapy for selected lesions. Its mechanisms of action are based on the internal change in hemodynamic parameters both in the main artery and within the aneurysm sac $(16,17)$. Also, the indentations along the stent act as a nidus for endothelial cell growth in the neck of the aneurysm that results in exclusion of the aneurysm from the circulation. In our study, a total of 19 patients were implanted with a FDS (15 saccular, 4 fusiform aneurysms). Antiplatelet therapy was started in all patients before the procedure. As a complication, one patient was admitted due to intraparenchymal hematoma on the $15^{\text {th }}$ postoperative day and died due to secondary infections whereas another patient had hemiparesis due to loss of feeder branches. Remaining seventeen patients who received FDS treatment did not have any significant complication during the follow-up period. 
Long-term follow-up after endovascular treatment is important to evaluate the stability of the treatment and to detect early recurrence. In many studies, it has been reported that at least two controls should be performed within the first year after endovascular treatment (4). Although DSA is the gold standard diagnostic method for the follow-up of these patients, it is a more invasive and expensive procedure compared to MRA. We performed the first control after the procedure in our center in the $3^{\text {rd }}$ month by the DSA. However, the patient was advised to have an earlier control visit in case any neurologic symptom developed or the aneurysm neck was not completely occluded in the first intervention.

The limitations of our study were small number of patients and limited follow-up period. Prospective studies should be conducted to determine the best treatment approach in different subtypes, localizations and clinical scenarios.

\section{Conclusion}

IAs can be effectively treated with endovascular techniques since cure rates are high and complications rates are reasonably low. Coil, FDS or both can be used in selected cases of ruptured or unruptured aneurysms. Wide neck aneurysms constitute a technical challenge for the radiologists and have worse prognosis regarding to recurrence and need for additional therapy.

\section{Ethics}

Ethics Committee Approval: Local Ethics Committee of University of Health Sciences Turkey, Gülhane Training and Research Hospital approved the study protocol (approval number: 2020-59) in accordance with the principles of the Declaration of Helsinki.

Informed Consent: Retrospective study.

Peer-review: Externally and internally peer-reviewed.

\section{Authorship Contributions}

Surgical and Medical Practices: G.Y., Concept: G.Y., M.T., Design: M.T., Data Collection or Processing: G.Y., Analysis or Interpretation: G.Y., M.T., Literature Search: G.Y., Writing: G.Y.

Conflict of Interest: No conflict of interest was declared by the authors.

Financial Disclosure: The authors declared that this study received no financial support.

\section{References}

1. Guglielmi G, Vinuela F, Dion J, Duckwiler G. Electrothrombosis of saccular aneurysms via an endovascular approach. Part 2: preliminary clinical experience. J Neurosurg. 1991;75:8-14.

2. Molyneux A, Kerr R, Stratton I, et al. International Subarachnoid Aneurysm Trial (ISAT) of neurosurgical clipping versus endovascular coiling in 2143 patients with ruptured intracranial aneurysms: a randomised trial. Lancet 2002;360:1267-1274.

3. Cognard C, Weill A, Castaings L, Rey A, Moret J. Intracranial berry aneurysms: angiographic and clinical results after endovascular treatment. Radiology. 1998;206:499-510.

4. Cognard C, Weill A, Spelle L, et al. Long-term angiographic follow-up of 169 intracranial berry aneurysms occluded with detachable coils. Radiology. 1999;62:348-356.

5. Zhang Y, Chong W, Qian Y. Investigation of intracranial aneurysm hemodynamics following flow diverter stent treatment. Med Eng Phys 2013;35:608-615.

6. Lylyk P, Miranda C, Ceratto R, et al. Curative endovascular reconstruction of cerebral aneurysms with the pipeline embolization device: the Buenos Aires experience. Neurosurgery. 2009;64:632-642.

7. Meyer FB, Morita A, Puumala MR, Nichols DA. Medical and surgical management of intracranial aneurysms. Mayo Clin proc. 1995;70:153-172.

8. Mohr JP. Stroke: Pathophysiology, Diagnosis, and Management. Churchill Livingstone;2004.

9. Zhao J, Lin H, Summers R, Yang M, Cousins BG, Tsui J. Current Treatment Strategies for Intracranial Aneurysms: An Overview. J Angiology. 2018;69:17-30.

10. Gawlitza M, Soize S, Barbe C, et al. Aneurysm Characteristics, Study Population, and Endovascular Techniques for the Treatment of Intracranial Aneurysms in a Large, Prospective, Multicenter Cohort: Results of the Analysis of Recanalization After Endovascular Treatment of Intracranial Aneurysm Study. AJNR Am J Neuroradiol. 2019;40:517-523.

11. Ovül I. Subarachnoid Hemorrhage (SAH). In: Altınors N, Baykaner K, Sekerci Z, Ozyurt E, Caner H, eds. Basic Neurosurgery, Ankara;1997:1-18.

12. Jagadeesan BD, Siddiq F, Grande AW, Tummala RP. Modified balloon assisted coil embolization for the treatment of intracranial and cervical arterial aneurysms using coaxial dual lumen balloon microcatheters: initial experience. J Neurointerv Surg. 2014;6:704-707.

13. Pukenas B, Albuquerque FC, Weigele JB, Hurst RW, Stiefel MF. Use of a New Double-Lumen Balloon Catheter for Single-Catheter Balloon-Assisted Coil Embolization of Intracranial Aneurysms: Technical Note. Neurosurgery. 2011;69(1 Suppl Operative):8-12

14. Ihn YK, Shin SH, Baik SK, Choi IS. Complications ofendovascular treatment for intracranial aneurysms: Management and prevention. Interv Neuroradiol. 2018;24:237-245.

15. Raymond J, Guilbert F, Weill A, et al. Long-term angiographic recurrences after selective endovascular treatment of aneurysms with detachable coils. Stroke 2003;34:1398-1403. 
16. Ravindran K, Casabella AM, Cebral J, Brinjikli W, Kallmes DF, Kadirvel R. Mechanism of Action and Biology of Flow Diverters in the Treatment of Intracranial Aneurysms. Neurosurgery 2020;86(Suppl 1):13-19.
17. Ravindran K, Salem MM, Alturki AY, Thomas AJ, Ogilvy CS, Moore JM. Endothelialization following flow diversion for intracranial aneurysms: a systematic review. AJNR Am J Neuroradiol. 2019;40:295-301. 